\title{
Blind estimation of a ship's relative wave heading
}

\author{
Nielsen, Ulrik Dam; Iseki, Toshio
}

Published in:

Proceedings of the 31st International Conference on Ocean, Offshore and Arctic Engineering

Publication date:

2012

Link back to DTU Orbit

Citation (APA):

Nielsen, U. D., \& Iseki, T. (2012). Blind estimation of a ship's relative wave heading. In Proceedings of the 31 st International Conference on Ocean, Offshore and Arctic Engineering (pp. OMAE2012-83305). American Society of Mechanical Engineers.

\section{General rights}

Copyright and moral rights for the publications made accessible in the public portal are retained by the authors and/or other copyright owners and it is a condition of accessing publications that users recognise and abide by the legal requirements associated with these rights.

- Users may download and print one copy of any publication from the public portal for the purpose of private study or research.

- You may not further distribute the material or use it for any profit-making activity or commercial gain

- You may freely distribute the URL identifying the publication in the public portal

If you believe that this document breaches copyright please contact us providing details, and we will remove access to the work immediately and investigate your claim 
Proceedings of the 31st International Conference on Ocean, Offshore and Arctic Engineering
OMAE 2012
July 1-6, 2012, Rio de Janeiro, Brazil

OMAE2012-83305

\section{BLIND ESTIMATION OF A SHIP'S RELATIVE WAVE HEADING}

\author{
Ulrik D. Nielsen \\ Department of Mechanical Engineering \\ Technical University of Denmark \\ 2800 Kgs. Lyngby, Denmark \\ Email: udn@mek.dtu.dk
}

\author{
Toshio Iseki \\ Department of Marine System Engineering \\ Tokyo University of Marine Science and Technology \\ Tokyo, 135-8533, Japan \\ Email: iseki@kaiyodai.ac.jp
}

\begin{abstract}
This article proposes a method to estimate a ship's relative heading against the waves. The procedure relies purely on shipboard measurements of global responses such as motion components, accelerations and the bending moment amidships. There is no particular (mathematical) model connected to the estimate, and therefore it is called a 'blind estimate'. The approach is in this introductory study tested by analysing simulated data. The analysis reveals that it is possible to estimate a ship's relative heading on the basis of shipboard measurements only.
\end{abstract}

\section{INTRODUCTION}

Knowledge about the sea state parameters at a ship's position in the ocean is fundamental input to operator guidance systems for ship safety and to performance monitoring systems used to increase fuel efficiency. There exists a substantial amount of work dealing with the estimation of directional wave spectra from shipboard measurements, where an analogy to traditional wave rider buoys is made, e.g., Iseki and Ohtsu [4], Nielsen [11], Nielsen and Iseki [12] and Nielsen and Stredulinsky [13] to mention but a few. From a directional wave spectrum estimate any sea state parameter can be obtained, including the mean wave direction or, equivalently, the mean relative wave heading. The wave buoy analogy provides typically wave estimates in fair agreement with other 'wave measuring devices' such as wave radar systems and wave rider buoys [13].

The wave buoy analogy relies completely on having an accurate ship hydrodynamic model from which transfer functions can be derived. It is clear that any additional information that can be obtained to either improve the estimate from the wave buoy analogy or to verify its findings is useful. Specifically, in recent developments made by Andersen and Storhaug [2] it will be valuable to have an (initial) estimate of the relative wave heading. This study looks at the possibility to estimate an advancing ship's mean relative wave heading by processing shipboard responses such as the wave-induced vertical acceleration and the roll and pitch angles. The estimate is made without any hydrodynamic model for what reason it is called a "blind estimate". Basically, the estimation process builds on calculations of ratios formed by spectral moments. The idea (or hypothesis) is that ratios of characteristic spectral parameters, formed by, say, the ratio between the standard deviations of the roll angle and the pitch angle, respectively, can be used to (qualitatively) estimate the mean relative heading of the ship. In other words, the hypothesis is that ranges can be specified for the ratios of spectral parameters, so that a value within the particular range indicates a relative heading in a limited interval on a scale from head sea to following sea.

The study looks at numerically generated ship response data only. Thus, a comprehensive set of time histories simulations of ship responses are processed. The responses are generated from short-crested wave scenarios represented by (unimodal) directional wave spectra.

The background of the hypothesis made towards making a blind estimate of a ship's relative heading relies on fundamentals within wave-ship interactions. However, no exact theory has been developed for the purpose. Instead, the following section briefly sketches the thoughts which lie behind the hypothesis. In the remaining part of the paper, it should be noted that when 
concern is given to wave parameters - wave height, period and direction - describing the sea state it is always understood that certain characteristic values are considered; e.g. 'significant wave height', 'zero-upcrossing period', and 'mean direction'.

\section{HYPOTHESIS - WAVE-SHIP INTERACTIONS}

The motions and structural responses of an advancing ship in a seaway depend on a vast number of factors. It is therefore a highly complicated task to predict/estimate about future waveship interactions. However, any ship has a certain characteristic response, or behaviour, to a given wave scenario, where the scenario is typically described by wave parameters such as zeroupcrossing period, significant height and mean direction. In theoretical calculations, the characteristic behaviour can be given in terms of, e.g., a vessel frequency response function, i.e. a transfer function, calculated for any desired motion or other type of response.

The transfer function of a particular response exhibits properties that (typically) change as the wave parameters change. This means that spectral calculations, where transfer functions are matched with a wave spectrum [7], in general lead to different outcomes depending on the wave parameters. This is illustrated in Figure 1 by Lloyd [7], that shows the effect of varying the peak period, keeping the significant wave height fixed, of the wave energy spectrum on the standard deviation of the relative motion for the forefoot of a frigate in irregular head waves. If attention is given only to the standard deviation $\sigma_{0}$ it is from this example clear that, under general conditions, nothing can be said about the values of the wave parameters. This is so because the

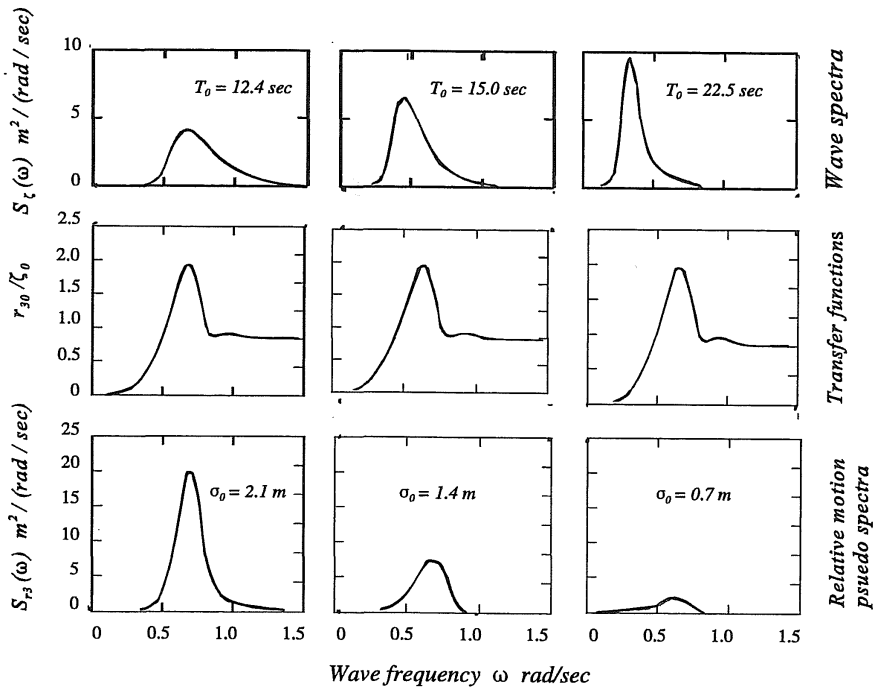

FIGURE 1. Effect of matching the wave energy spectrum with the transfer function. Relative motions at the bow of the frigate at 20 knots in irregular head waves: Significant wave height $5.5 \mathrm{~m}$. Lloyd [7] situation at the right-hand side columns, where $\sigma_{0}=0.7 \mathrm{~m}$, could just as well have been obtained in the situation shown at the lefthand side columns if the wave spectrum were down-scaled (reducing $H_{s}$ ). However, if several responses were considered at the same time it could be differently, especially if ratios - instead of absolute values - were formed of the standard deviations for the considered responses. In such a case it could be that one of the ratios, say, $r_{i j}=\frac{\sigma_{i}}{\sigma_{j}}$ would take a, more or less, constant value for changing (zero-upcrossing) period and wave height but keeping the relative wave heading fixed. Furthermore, if some (larger) fixed change in the relative heading implies a shift to a different but constant value of the ratio $r_{i j}$, independent of changes in significant wave height and zero-upcrossing period, this observation could be used to make a qualitative estimate of the relative heading. The idea is illustrated in the following hypothetical example.

\subsection{A Hypothetical Example}

Two responses, say, 1 and 2, are measured on a ship that advances in a moderate sea state with a given (unknown) significant wave height. From statistical analysis it is found that the ratio between the standard deviations of the two responses is $r_{12}=4.2$ at a given time based on the past 15 minutes of measurements. On the other hand, numerical simulations of the two responses have been conducted for the same ship and, based on the simulations, the diagram presented in Figure 2 has been obtained. The numerical simulations have been made for a significant wave height not too different from the one representing the "real" sea state. Moreover, the simulations are made for five different wave periods $\left(T_{1}, T_{2}, \ldots, T_{5}\right)$, representing a reasonable interval and, finally, five different relative headings are consid-

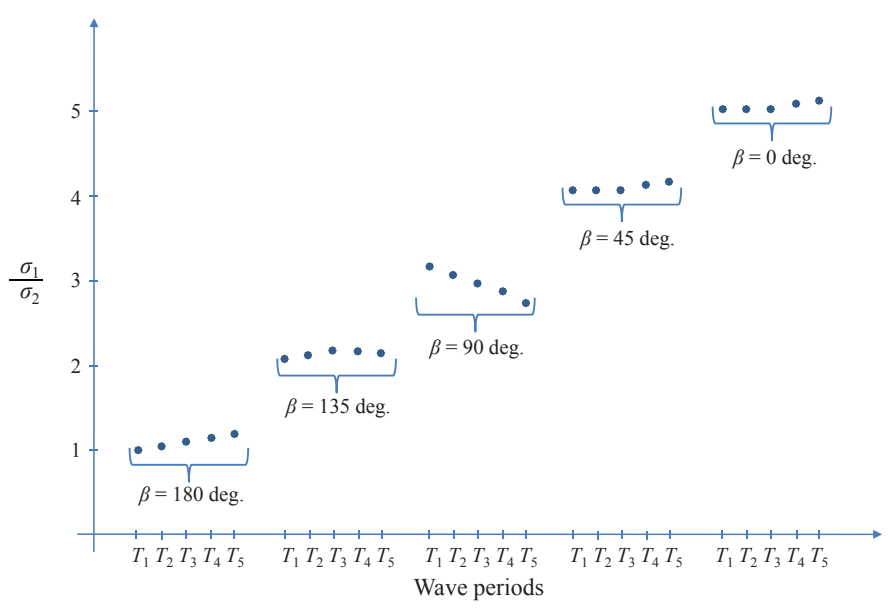

FIGURE 2. Ratios of standard deviations obtained from (hypothetical) simulations made for five different headings each carried out for five different wave periods $\left(T_{1}, T_{2}, \ldots, T_{5}\right)$. 
ered for each combination of wave periods and significant wave height. From Figure 2, it is seen that the ratio between $\sigma_{1}$ and $\sigma_{2}$ takes a value close to 3 for $T_{3}$ when $\beta=90 \mathrm{deg}$. and, similarly, the ratio is around 5 when $\beta=0 \mathrm{deg}$. independent of the wave period. It is clear that the inherent stochastic behaviour of the given ratio should be introduced. Hence, each point in the diagram has been attained as the mean value calculated from, say, 10 sets of simulations of the responses 1 and 2 .

The measured ratio of standard deviations was $\frac{\sigma_{1}}{\sigma_{2}}=4.2$, so in this hypothetical example it seems reasonable to assume the relative wave heading to be around $45 \mathrm{deg}$.

The diagram in Figure 2 has been constructed to illustrate the hypothesis cast forward in this study. The central point is to realise that the ratio of, say, the standard deviations has to take rather distinct and (preferably) constant values for different headings irrespectively on the values of wave height and period. If the 5 times 5 points in the diagram had all been aligned on a, more or less, horizontal line, it would not be possible to say anything about the relative wave heading from a measured ratio of the considered standard deviations. Neither should points corresponding to two (or more) 'neighboring' relative headings - overlap each other on the ordinate axis, since this would also make it impossible to estimate the relative heading on the basis of a measured ratio of standard deviations. However, additional information can be considered by taking into account other ratios derived from spectral moments. Moreover, pairs of several responses can be used to construct diagrams like the one in Figure 2. Consequently, the analysis made later will be based on more than two responses and on different spectral ratios.

It is important to note that the numerical simulations are made for one 'characteristic' significant wave height only. The reason is that the analysis is assumed to be independent on the significant wave height, since ratios - and not absolute values of spectral parameters are considered.

\section{CROSS SPECTRAL ANALYSIS}

The response measurements carried out on a ship are obtained as time histories. However, to facilitate an efficient calculation of characteristic (statistical) parameters the time histories can be transformed into the frequency domain by cross spectral analysis. Various tools and techniques are available for this analysis, where direct application of fast Fourier transformation (FFT) is widely used. More elaborate procedures exist, however, in terms of multivariate autoregressive (MVAR) modelling, see Akaike and Nakagawa [1], by application of, e.g., the stepwise least squares algorithm $[9,16]$ for the determination of the MVAR coefficients. The use of MVAR modelling facilitates an automatic determination of the "optimum" response spectra, which is often advantageous compared to standard FFT, where manual choices on window sizing etc. have an influence on the values of the individual spectral components. In some studies, like for ex-
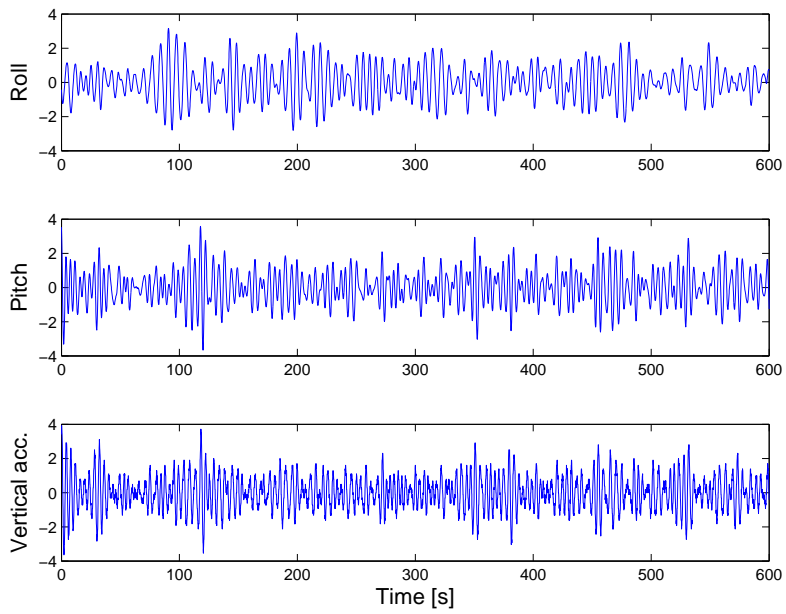

FIGURE 3. Three responses: pitch, roll, and vertical acceleration at FP measured on TS Shioji-maru. The individual response has been normalised with its standard deviation.

ample the wave buoy analogy $[4,11]$, any 'manual influence' is considered as a negative property. For this reason, MVAR modelling is typically applied to do the spectral analysis and so is the case in this study.

Figure 3 shows a set of three time histories measured on the training ship Shioji-Maru belonging to Tokyo University of Marine Science and Technology. The shown time histories are roll and pitch, both measured at a position close to COG, and vertical acceleration at FP. Cross spectral analysis can be performed by application of MVAR modelling and the outcome is seen in Figure 4 , where it is noted that the spectra depend on the encounter
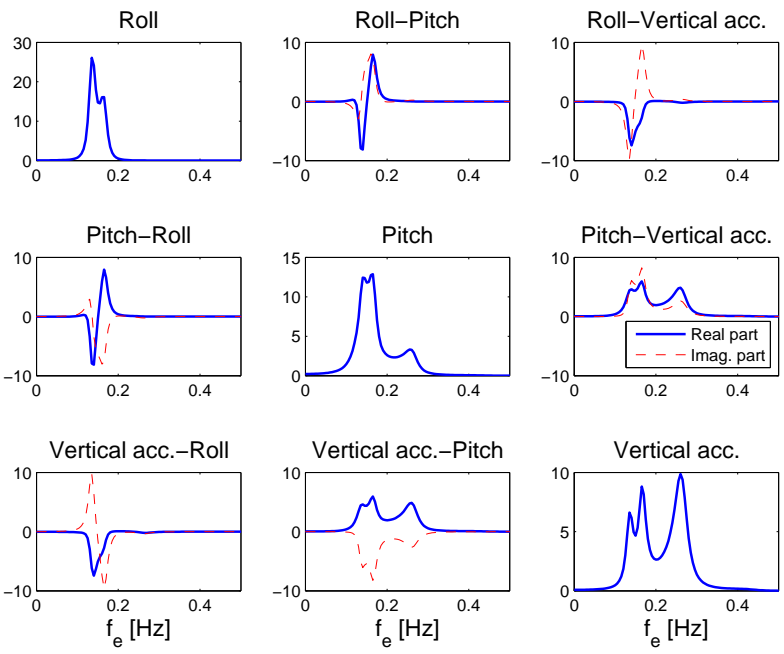

FIGURE 4. The non-dimensionalised cross spectra associated to the time histories shown in Figure 3. 
frequency $f_{e}$. The analysis of three responses leads to nine $(3 \mathrm{x}$ 3 ) spectra and it can be seen that the off-diagonal elements are pairwise complex conjugated. The off-diagonal spectra provide information about the correlation between a given combination of two of the considered responses. This means that the response spectrum, $S_{i j}\left(f_{e}\right)$, of the $i$-th and $j$-th responses can be used to measure the linear correlation between the two responses at each frequency [8]. In signal processing this measure is called the mean squared coherence (MSC) and it is directly analogous to the squared correlation coefficient in linear regression [15].

In this introductory study on blind estimation of a ship's relative heading the off-diagonal spectra will (basically) not be considered, although it is likely that valuable information can be provided by the analysis of, say, the MSC. However, presently an exact methodology has not yet been developed for this purpose.

The 'true physical' response spectra, sometimes also denoted the power spectral densities [15], are the diagonal elements in Figure 4. From any one of the response spectra, $S_{R}\left(f_{e}\right)$, the $n$ th order spectral moment can be calculated

$$
m_{n, R}=\int_{0}^{\infty} S_{R}\left(f_{e}\right) f_{e}^{n} d f_{e}, \quad n=0,1,2,3,4
$$

where, for example, the standard deviation $\sigma_{R}$ of the considered response is obtained directly as the square root of the 0 -th order moment $m_{0, R}$. Other interesting measures are the mean pe$\operatorname{riod} T_{R, m}$, the (mean) zero-upcrossing period $T_{R, z}$, and the (mean) peak period $T_{R, p}$ that can all be obtained by use of spectral moments:

$$
\begin{gathered}
T_{R, m}=\frac{m_{0}}{m_{1}} \\
T_{R, z}=\sqrt{\frac{m_{0}}{m_{2}}} \\
T_{R, p}=\sqrt{\frac{m_{2}}{m_{4}}}
\end{gathered}
$$

\section{GENERATION OF TIME HISTORIES}

If a linear relationship between ship responses and wave excitations is assumed, the time history of a response $R(t)$ measured on a ship can be expressed in terms of a complex-valued frequency response function $\Phi_{R}(\omega, \beta)$ and a directional wave spectrum $E\left(\omega_{n}, \beta_{m}\right)$, where $\omega$ and $\beta$ are the wave frequency and the wave direction, respectively. Traditionally, wave records are assumed to be Gaussian processes and to secure a Gaussian response the time series representation by Jensen and Capul [6] is applied. Thus, the response is determined by a set of uncorre- lated, standard normal distributed variables $u_{m n}$ and $\bar{u}_{m n}$. Hence,

$$
R(t)=\sum_{n=1}^{N_{0}} \sum_{m=1}^{M_{0}}\left[u_{m n} c_{m n}(t)+\bar{u}_{m n} \bar{c}_{m n}(t)\right]
$$

The deterministic coefficients $c_{m n}(t)$ and $\bar{c}_{m n}(t)$ are given by

$$
\begin{gathered}
c_{m n}(t)=\sigma_{m n}\left|\Phi_{R}\left(\omega_{n}, \beta_{m}\right)\right| \cos \left(\omega_{e, n} t+\varepsilon_{m n}\right) \\
\bar{c}_{m n}(t)=-\sigma_{m n}\left|\Phi_{R}\left(\omega_{n}, \beta_{m}\right)\right| \sin \left(\omega_{e, n} t+\varepsilon_{m n}\right) \\
\sigma_{m n}^{2}=E\left(\omega_{n}, \beta_{m}\right) \Delta \omega_{n} \Delta \beta_{m}
\end{gathered}
$$

where the directional wave spectrum has been discretised into a number of wave frequencies $N_{0}$ and headings $M_{0} . \Delta \omega_{n}$ and $\Delta \beta_{m}$ are the increments of the discrete wave frequencies and the discrete headings, respectively. The phase angles are calculated from

$$
\tan \left(\varepsilon_{m n}\right)=\frac{\operatorname{Im}\left[\Phi_{\mathrm{R}}\left(\omega_{\mathrm{n}}, \beta_{\mathrm{m}}\right)\right]}{\operatorname{Re}\left[\Phi_{\mathrm{R}}\left(\omega_{\mathrm{n}}, \beta_{\mathrm{m}}\right)\right]}
$$

It should be noted that white noise (uniform, zero mean) has been added to the synthetic data to make the situation more realistic. The maximum signal-to-noise ratio is chosen to 10 , and this value has been set completely subjectively with no reference to datasheets of sensor manufacturers (because of lack of specifications by the manufacturers, see also Pascoal and Guedes Soares [14]). The white noise has been added with a sampling rate of $10 \mathrm{~Hz}$, after which smoothing has been applied.

\subsection{Vessel and Test Cases}

Ship motion time histories have been generated for the training ship (T.S.) Shioji-maru of Tokyo University of Marine Science and Technology. A photo and principal particulars of the

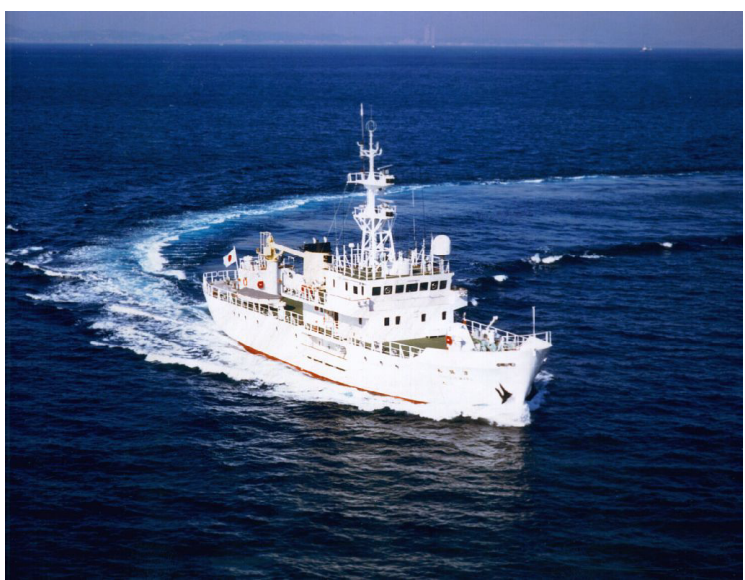

FIGURE 5. T.S. Shioji-maru used in the study. 
TABLE 1. Principal particulars of T.S. Shioji-maru.

\begin{tabular}{lr} 
Length, $L_{\mathrm{pp}}$ & $46.00 \mathrm{~m}$ \\
Breadth, $B_{\mathrm{mld}}$ & $10.00 \mathrm{~m}$ \\
Depth, $D_{\mathrm{mld}}$ & $6.10 \mathrm{~m}$ \\
Draught, $T_{\mathrm{mld}}$ & $2.65 \mathrm{~m}$ \\
Displacement & $659.4 \mathrm{t}$ \\
\hline
\end{tabular}

ship are shown in Figure 5 and Table 1, respectively. In this study, the simulated motions are roll, pitch and vertical acceleration. The former two motions (roll, pitch) relate to the centre of gravity of the ship whereas the vertical acceleration is given at a position close to the forward perpendicular.

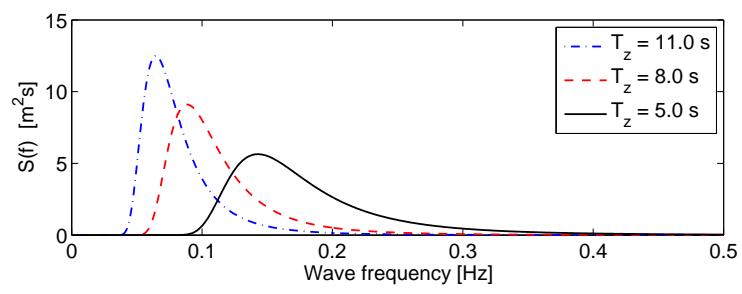

FIGURE 6. Plots of wave spectra obtained from a JONSWAP spectrum with $H_{s}=3.0 \mathrm{~m}$ and $T_{z}=\{11.0,8.0,5.0\} \mathrm{s}$.
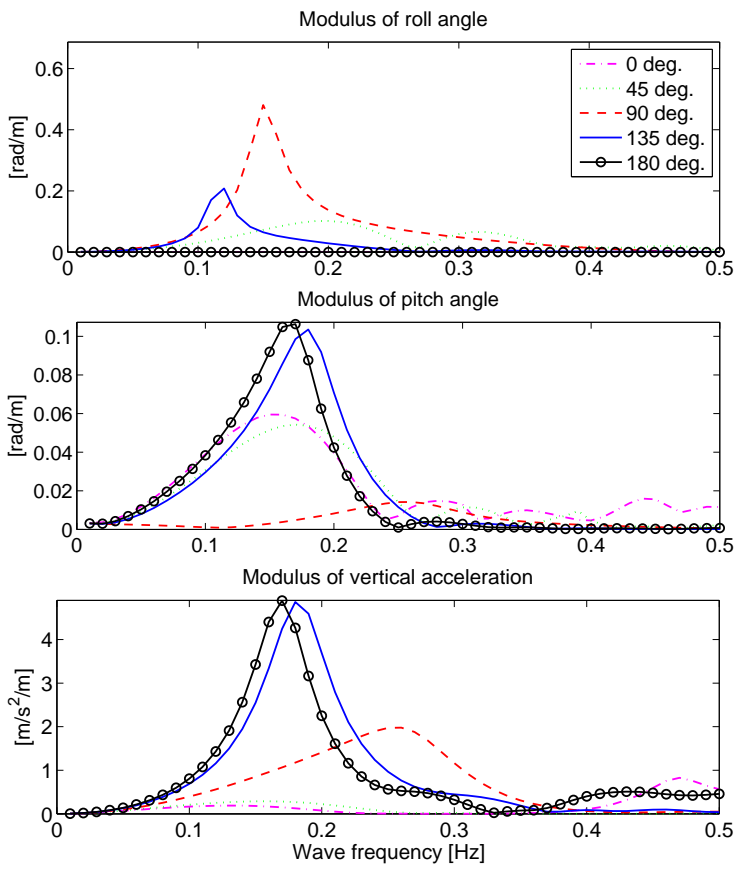

FIGURE 7. Modulus of transfer functions; the roll angle (upper plot), the pitch angle (middle plot) and the vertical acceleration at FP (lower plot).
TABLE 2. The underlying wave parameters of the test cases. Note that $\beta=180$ deg. is head sea.

\begin{tabular}{lccc}
\hline Cases & $H_{s}[\mathrm{~m}]$ & $\beta$ [deg.] & $T_{z}[\mathrm{~s}]$ \\
\hline$\{\mathrm{A} 1, \mathrm{~A} 2, \ldots, \mathrm{A} 5\}$ & 3.0 & 180 & $\{11.0 ; 9.5 ; 8.0 ; 6.5 ; 5.0\}$ \\
$\{\mathrm{B} 1, \mathrm{~B} 2, \ldots, \mathrm{B} 5\}$ & 3.0 & 135 & $\{11.0 ; 9.5 ; 8.0 ; 6.5 ; 5.0\}$ \\
$\{\mathrm{C} 1, \mathrm{C} 2, \ldots, \mathrm{C} 5\}$ & 3.0 & 90 & $\{11.0 ; 9.5 ; 8.0 ; 6.5 ; 5.0\}$ \\
$\{\mathrm{D} 1, \mathrm{D} 2, \ldots, \mathrm{D} 5\}$ & 3.0 & 45 & $\{11.0 ; 9.5 ; 8.0 ; 6.5 ; 5.0\}$ \\
$\{\mathrm{E} 1, \mathrm{E} 2, \ldots, \mathrm{E} 5\}$ & 3.0 & 0 & $\{11.0 ; 9.5 ; 8.0 ; 6.5 ; 5.0\}$ \\
\hline
\end{tabular}

To limit the complexity of this introductory study it is decided to focus on test cases represented by short-crested waves obtained from unimodal wave spectra. This means that mixed sea conditions are not considered. The test cases are established from the sets of wave parameters seen in Table 2. The table provides
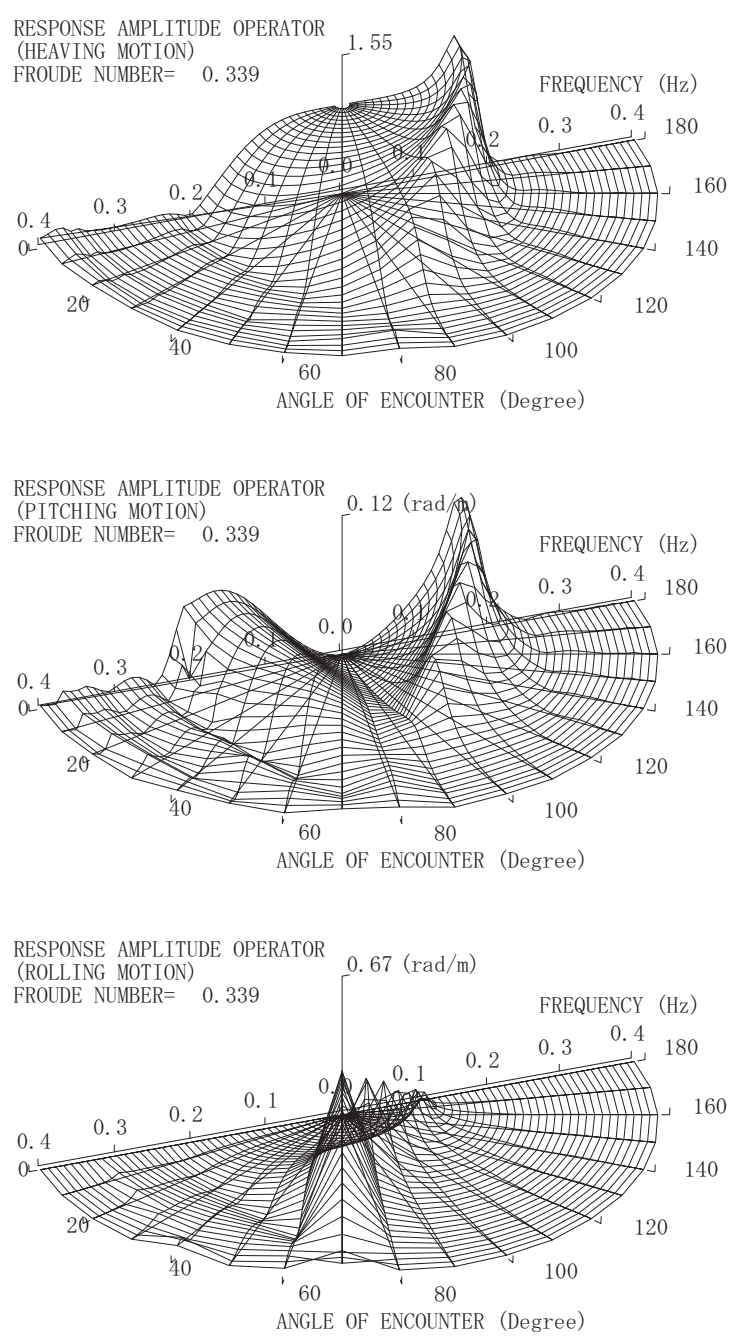

FIGURE 8. 3-D plots of transfer functions; heave, pitch and roll. 
the significant wave height $H_{s}$, the relative mean wave heading $\beta$ and the zero-upcrossing period $T_{z}$. It can be observed that not all combinations of wave height and period are realistic, since a sea state with $H_{s}=3.0 \mathrm{~m}$ and $T_{z}=5.0 \mathrm{~s}$ is very unlikely to practically occur.

The wave spectrum is modelled as a (directional) JONSWAP spectrum, including a frequency dependent spreading [12]. The variation with wave frequency is visualised in Figure 6 for the considered range of $T_{z}$. The range of $T_{z}$ is chosen to be representative to real ocean waves in which T.S. Shioji-maru typically operates. Based on the plots of the modulus of the individual transfer function, see Figures 7 and 8, for T.S. Shioji-maru it is seen that the chosen range of $T_{z}$ corresponds to waves which will excite the considered responses.

Examples of the motion time histories are shown in Figure 9 , where it is seen that one set of simulations covers a time span of 1,000 seconds. The upper plot shows the sea surface elevation amidships, while the three lower plots are the roll angle, the pitch angle and the vertical acceleration at FP, respectively. In the
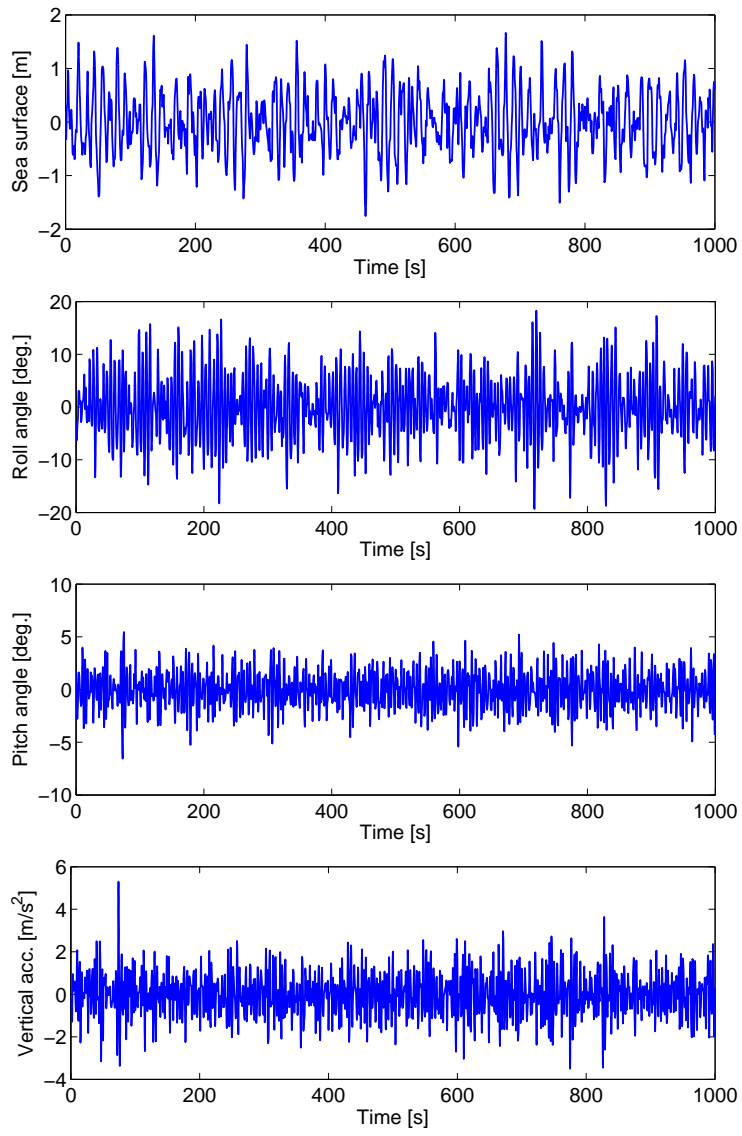

FIGURE 9. Time history simulations of the sea surface elevation, the roll angle, the pitch angle, and the vertical acceleration at FP, respectively, using wave scenario B3. analysis that follows, it is important to mention that each wave scenario (A1, A2, ..., C2, C3, .., E5) is used to generate 10 sets of time histories. Thus, statistics can be introduced in the analysis. The considered set of time history simulations in Figure 9 corresponds to one outcome using wave scenario B3.

\section{RESULTS AND DISCUSSION}

Figure 10 shows four plots that illustrate the behaviour of different spectral ratios. Ratios are formed between the standard deviation $\sigma_{R}$, the mean response period $T_{R, m}$, the zero-upcrossing response period $T_{R, z}$, and the peak response period $T_{R, p}$. Each ratio is calculated for three different combinations of responses, where the combinations are 'roll angle over pitch angle', 'pitch angle over vertical acceleration', and 'roll angle over vertical ac-
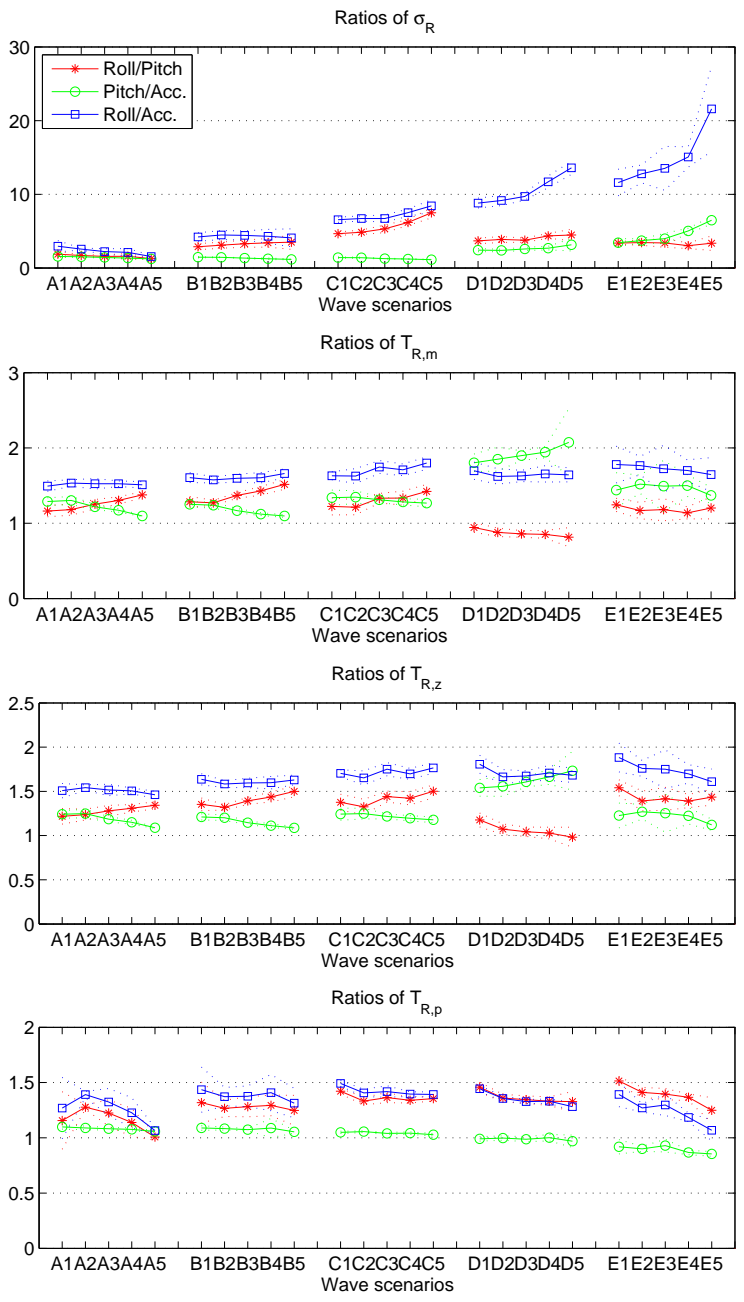

FIGURE 10. Ratios derived from spectral moments; cf. Eqs. (1)-(4). Each node corresponds to the mean value obtained from 10 simulations; the statistical spreading is included as thin dotted lines. 
celeration', respectively. In any case, the ratios are shown for the 5 times 5 wave scenarios $(\mathrm{A} 1, \mathrm{~A} 2, \ldots, \mathrm{C} 2, \mathrm{C} 3, \ldots, \mathrm{E} 5)$ and it should be noted that a specific value of a given ratio is obtained as the mean value based on ten simulations as explained previously. Moreover, the statistical spreading to the specific value is included by plotting also a value obtained as the mean value plus and minus, respectively, the standard deviation. The statistical spreading is shown by the thin dotted lines. In general, it is observed that there is a rather small spreading around the mean values for most ratios at the different wave scenarios. However, it is seen that the ratio of standard deviations of roll and vertical acceleration has quite some spreading for wave scenarios E1, E2, ..., E5. The statistical spreading around each node has a consequence for the limiting interval of the particular ratio for a given set of wave scenarios. For example, the limiting interval of the ratio of standard deviations of roll and vertical acceleration is found as $[9.79 ; 27.4]$ for wave scenarios E1, E2, ..., E5.

Independent on the considered plot in Figure 10, it appears evident that a situation, as indicated in the hypothetical example (Figure 2), does not appear. In the following, focus will be on the ratios of standard deviations; the upper plot in Figure 10. In particular, it is noted that the ratio formed by 'roll angle over vertical acceleration' has some resemblance to the diagram of the hypothetical example. However, if the statistical spreading is taken into account, there are (small) overlaps between more of the sets of wave scenarios (A1, A2, ..., C2, C3, ..., E5). This observation is confirmed by Table 3, where the limiting interval for each ratio is given for all wave scenarios. It is noted that Table 3 contains the results of all response combinations as well as the results of all spectral ratios. The table reveals, for example, that if the ratio of standard deviations of the roll angle and the vertical acceleration is less than 5.30, the relative wave heading cannot be that of wave scenarios C, D, or E corresponding to beam sea, stern quartering sea and following sea, respectively. In other words, this means that the ship sails in either head sea or bow quartering sea. However, if the relative wave heading were to be based on the ratio of standard deviations of the roll angle and the vertical acceleration alone, it cannot be said which of the two headings - head sea or bow quartering sea - is the correct one, since part of the limiting interval $[1.40 ; 3.71]$ of wave scenarios $\mathrm{A} 1, \mathrm{~A} 2, \ldots$, A5 is located inside the limiting interval $[2.88 ; 5.30]$ of scenarios B1, B2,..., B5. The answer is, on the other hand, given directly by considering the ratio of standard deviations of the roll angle and the pitch angle, because of a distinct separation between the limiting intervals of this ratio in the given cases. Thus, the intervals are $[1.16 ; 2.28]$ and $[2.48 ; 4.46]$. As a final confirmation to answer which heading is the correct one, it should be noted that the limiting interval of the ratio formed by the standard deviations of the pitch angle and the vertical acceleration does not overlap any of the two other limiting intervals for wave scenarios B1, B2, ..., B5, which is not the case for wave scenarios A1, $\mathrm{A} 2, \ldots, \mathrm{A} 5$. In summary, it has been seen that it is possible to
TABLE 3. The limiting intervals on all spectral ratios for the different sets of wave scenarios.

\begin{tabular}{|c|c|c|c|}
\hline Cases & Roll/Pitch & Pitch/Acc. & Roll/Acc. \\
\hline \multicolumn{4}{|c|}{ Ratios of $\sigma_{R}$} \\
\hline A & {$[1.16 ; 2.28]$} & {$[1.19 ; 1.67]$} & {$[1.40 ; 3.71]$} \\
\hline B & {$[2.48 ; 4.46]$} & {$[1.12 ; 1.54]$} & {$[2.88 ; 5.30]$} \\
\hline $\mathrm{C}$ & {$[4.44 ; 8.17]$} & {$[1.09 ; 1.49]$} & {$[6.20 ; 9.27]$} \\
\hline $\mathrm{D}$ & {$[3.29 ; 5.26]$} & {$[2.20 ; 3.99]$} & {$[8.14 ; 14.5]$} \\
\hline $\mathrm{E}$ & {$[2.38 ; 4.36]$} & {$[2.89 ; 6.97]$} & {$[9.79 ; 27.4]$} \\
\hline \multicolumn{4}{|c|}{ Ratios of $T_{R, m}$} \\
\hline A & {$[1.09 ; 1.42]$} & {$[1.09 ; 1.36]$} & {$[1.42 ; 1.59]$} \\
\hline B & {$[1.21 ; 1.58]$} & {$[1.08 ; 1.29]$} & {$[1.53 ; 1.72]$} \\
\hline $\mathrm{C}$ & {$[1.11 ; 1.52]$} & {$[1.21 ; 1.44]$} & {$[1.52 ; 1.88]$} \\
\hline D & {$[0.69 ; 1.01]$} & {$[1.63 ; 2.52]$} & {$[1.52 ; 1.81]$} \\
\hline $\mathrm{E}$ & {$[1.03 ; 1.35]$} & {$[1.15 ; 1.84]$} & {$[1.41 ; 2.04]$} \\
\hline \multicolumn{4}{|c|}{ Ratios of $T_{R, z}$} \\
\hline A & {$[1.13 ; 1.38]$} & {$[1.08 ; 1.30]$} & {$[1.42 ; 1.59]$} \\
\hline B & {$[1.28 ; 1.56]$} & {$[1.07 ; 1.25]$} & {$[1.53 ; 1.70]$} \\
\hline $\mathrm{C}$ & {$[1.24 ; 1.57]$} & {$[1.15 ; 1.31]$} & {$[1.57 ; 1.83]$} \\
\hline $\mathrm{D}$ & {$[0.87 ; 1.25]$} & {$[1.44 ; 1.95]$} & {$[1.58 ; 1.91]$} \\
\hline $\mathrm{E}$ & {$[1.28 ; 1.63]$} & {$[1.04 ; 1.47]$} & {$[1.47 ; 2.05]$} \\
\hline \multicolumn{4}{|c|}{ Ratios of $T_{R, p}$} \\
\hline A & {$[0.90 ; 1.42]$} & {$[1.05 ; 1.12]$} & {$[0.99 ; 1.55]$} \\
\hline B & {$[1.11 ; 1.50]$} & {$[1.00 ; 1.17]$} & {$[1.18 ; 1.64]$} \\
\hline $\mathrm{C}$ & {$[1.30 ; 1.45]$} & {$[1.02 ; 1.06]$} & {$[1.37 ; 1.52]$} \\
\hline D & {$[1.24 ; 1.50]$} & {$[0.91 ; 1.03]$} & {$[1.24 ; 1.47]$} \\
\hline $\mathrm{E}$ & {$[1.14 ; 1.55]$} & {$[0.83 ; 0.99]$} & {$[0.97 ; 1.50]$} \\
\hline
\end{tabular}

estimate whether a ship sails in head sea or bow quartering sea; and excluding the other headings beam sea, stern quartering sea, and following sea. On the contrary, the ship does not sail in head sea or stern quartering sea, if the ratio of standard deviations of the roll angle and the vertical acceleration is more than 6.20 , cf. Table 3. If so, it is possible to estimate the relative heading as well:

Beam sea will be experienced in case the ratio of standard deviations of the pitch angle and the vertical acceleration is in the limiting interval $[1.09 ; 1.49]$, corresponding to wave scenarios $\mathrm{C} 1, \mathrm{C} 2, \ldots, \mathrm{C} 5$. The reason is that this limiting interval is completely separated from the two other limiting intervals of the same ratio for the two other sets, D and E, of wave scenarios.

It is, however, not possible to estimate if the ship sails in stern quartering sea or following sea (excluding the other headings by means of exclusion) when only ratios of standard deviations are considered. In this case, it is necessary to consider also 
the ratio(s) of, e.g., the mean response periods and/or the zeroupcrossing periods. In either case, the ratios of the roll angle over the pitch angle and the pitch angle over the vertical acceleration, respectively, can be used. Thus, the limiting intervals of sets $\mathrm{D}$ and $\mathrm{E}$ are fully separated when the ratio(s) of the roll angle and the pitch angle are considered; for $T_{R, m}$ and $T_{R, z}$ the intervals are $[0.69 ; 1.01]$ versus $[1.03 ; 1.35]$ and $[0.87 ; 1.25]$ versus [1.28; 1.63], respectively. In addition, it is seen that the limiting intervals of the two response combinations are completely separated in wave scenarios D1, D2,..., D5, which is not the case in wave scenarios E1, E2, ..., E5.

In conclusion, it has been seen that it is possible to (qualitatively) estimate the mean relative wave heading from spectral ratios formed by different responses.

\subsection{Additional Information by the Off-diagonal Spec- tra}

It has been mentioned already that a (special) method has not been developed to take the off-diagonal spectra, cf. Figure 4, into account. On the other hand, it is possible to introduce the off-diagonal spectra in a similar manner as done for the ratio of standard deviations. Thus, the integral of the real part and the imaginary part, respectively, can be calculated using Eq. (1), for $n=0$, applied to any one combination of two responses using the cross spectrum $S_{i j}\left(f_{e}\right)$. Subsequently, the ratio of the two integral values - 'real value over imaginary value' - is formed, and in this way Figure 11 can be obtained. If details of the figure are studied, it is possible only to deduce something from the ratio considering the combination of the pitch angle and the vertical acceleration, since the two other ratios behave odd. The ratio of the combination of the pitch angle and the vertical acceleration, see Figure 12, is seen to exhibit an interesting property, as the ratio is negative for the sets, $\mathrm{A}, \mathrm{B}$, and $\mathrm{C}$, of wave scenarios corresponding to head, bow quartering and beam seas, whereas the ratio is positive for the two other sets, D and E. Obviously, this information is of high value, when a "blind estimate" of the heading is to be given. In the future, it should therefore be strived for to develop a consistent approach handling the off-diagonal

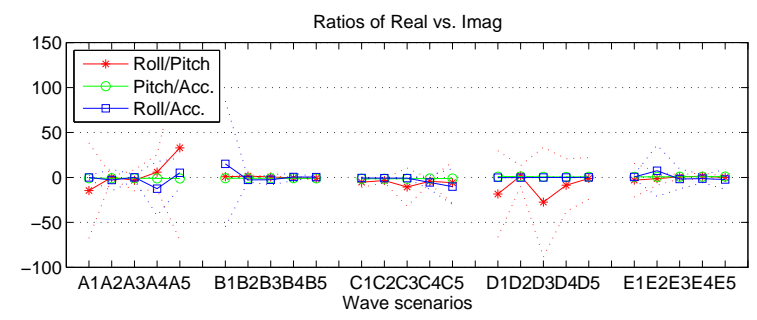

FIGURE 11. Ratios derived from Eq. (1) applied to the real part and the imaginary part, respectively, of a given combination of two responses.

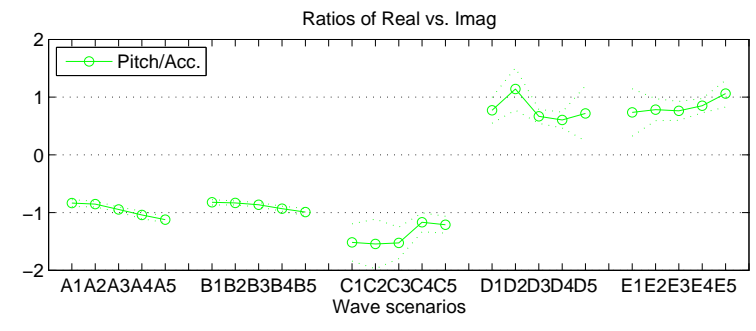

FIGURE 12. The plot is identical to Figure 11 but keeping only the combination of the pitch angle and the vertical acceleration.

spectra in a more refined manner; not only considering integral values.

\section{APPLICATION FOR REAL-TIME OPERATIONS}

This study is based on numerical simulations only, and based on the positive findings herein, an approach should be developed to handle real-time ship operations considering full-scale data. The procedure could be to make a comprehensive set of time series simulations for many different wave scenarios. In this way, a large database with information about spectral ratios for different responses could be produced; similar to what has been obtained in this paper's study. During actual ship operations, spectral ratios from full-scale data can be compared to information from the database and, thus, it will be possible to obtain a blind estimate of the ship's relative wave heading.

\section{SUMMARY AND CONCLUSIONS}

It has been shown that a (specific) ship's relative wave heading can be obtained from a 'blind' estimate using only a set of measured responses. This means that a mathemati$\mathrm{cal} /$ hydrodynamical model is not needed. There are some important aspects which should be noted and/or further investigated:

- In this study, numerical simulations have been considered only; the procedure/hypothesis must be confirmed by considering full-scale data.

- The study of ratios - and not absolute values - of spectral values is made to make the procedure independent to significant wave height. This (assumed) independency to significant wave height must be validated.

- A limited set of responses has been used in this study; can other responses like vertical bending moment amidships provide additional (valuable) information?

- How to (better) understand and utilise the off-diagonal spectra.

- It has been suggested to form a comprehensive database in order to apply the procedure to real-time operations. However, mixed sea conditions must be contained also in 
a database. Therefore, further studies like the one herein should be made for multimodal seas.

- A rather small and maybe 'characteristic'(?) vessel has been studied; what about other types of ships?

\subsection{A Note about Previous Work}

During the very finalisation of the present paper it has come to the first author's knowledge of a similar idea to make a heading estimation based on ship motion measurements. Thus, Davis et al. [3] devise an approximate and simple method for determining the mean wave direction in terms of the relative magnitudes of measured pitch and roll and vertical velocity of the vessel centre of gravity. Basically, the approach is based on calculations relating pitch and roll with the resultant angular slope of the deck and the resultant inclination [10]. It is found that, similarly to the present study, it seems possible to estimate the mean heading in sectors of $\pm 22.5 \mathrm{deg}$., see also Jacobi et al. [5]. As a work for the future, it will be interesting to combine the approach outlined in [3] with the ideas presented in the present paper.

\section{ACKNOWLEDGMENT}

During the processing of the paper, some of the discussions between the authors were made while the first author stayed at Tokyo University of Marine Science and Technology as part of the JSPS fellowship program (S11175). Sincere thanks are directed to the Japan Society for the Promotion of Science (JSPS) for the sponsorship of the stay taking place in January 2012.

\section{REFERENCES}

[1] H. Akaike and T. Nakagawa, Statistical Analysis and Control of Dynamic Systems, KTK Scientific Publishers, 1988.

[2] I. M. V. Andersen and G. Storhaug, Dynamic Selection of Ship Responses for Estimation of on-site Directional Wave Spectra, Proc. 31st OMAE (Rio de Janeiro, Brazil), 2012.

[3] M.R. Davis, N.L. Watson, and D.S. Holloway, Measurement of Response Amplitude Operators for an 86 m HighSpeed Catamaran, Journal of Ship Research 49 (2005), 121-143.

[4] T. Iseki and K. Ohtsu, Bayesian estimation of directional wave spectra based on ship motions, Control Engineering Practice 8 (2000), 215-219.

[5] G. Jacobi, G. Thomas, M.R. Davis, D.S. Holloway, G. Davidson, and T. Roberts, Full-scale motions of a large high-speed catamaran: The influence of wave environment, speed and ride control system, Submitted for possible publication in Trans. RINA (2012).

[6] J.J. Jensen and J. Capul, Extreme response predictions for jack-up units in second order stochastic waves by FORM, Probabilistic Engineering Mechanics 21 (2006), 330-338.
[7] A.R.J.M. Lloyd, Seakeeping, 2nd ed., Ellis Horwood, 1998.

[8] S.L. Marple, Digital Spectral Analysis with Applications, Prentice-Hall, 1987.

[9] A. Neumaier and T. Schneider, Estimation of Parameters and Eigenmodes of Multivariate Autoregressive Models, ACM Transactions on Mathematical Software 27 (2001), 27-57.

[10] J.N. Newman, Marine Hydrodynamics, MIT Press, 1977.

[11] U.D. Nielsen, Estimations of on-site directional wave spectra from measured ship responses, Marine Structures 19 (2006), 33-69.

[12] U.D. Nielsen and T. Iseki, A Study on Parametric Wave Estimation Based on Measured Ship Motions, Journal of Japan Institute of Navigation 126 (2012).

[13] U.D. Nielsen and D.C. Stredulinksy, Sea state estimation from an advancing ship - A comparative study using sea trial data, Applied Ocean Research 34 (2012), 33-44.

[14] R. Pascoal and C. Guedes Soares, Non-parametric wave spectral estimation using vessel motions, Applied Ocean Research 30 (2008), 46-53.

[15] W.D. Penny, Signal Processing Course, April 2000, Lecture notes, Course offered at the Department of Imaging Neuroscience, University College London.

[16] T. Schneider and A. Neumaier, Algorithm 808: ARfit A Matlab Package for the Estimation of Parameters and Eigenmodes of Multivariate Autoregressive Models, ACM Transactions on Mathematical Software 27 (2001), 58-65, http://www.gps.caltech.edu/ tapio/arfit/. 